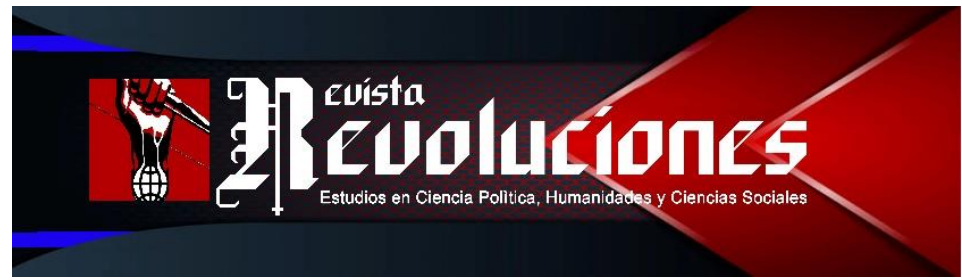

\title{
LA CONTINUIDAD DE LA PREVENCIÓN DE LA VIRUELA EN EL PERÚ: MEDIDAS DEL SUPERIOR GOBIERNO (1806-1820) AL GOBIERNO DEL PROTECTORADO (1822)
} \author{
(1822)

Arnaldo Mera ${ }^{1}$
PONTIFICIA UNIVERSIDAD CATÓLICA DEL PERÚ
PERU
arnaldo_mera@ @hotmail.com
https://orcid.org/oooo-ooo1-9331-7776

The continuity of the prevention of smallpox in Peru: measures of the Superior Government (1806-1820) to the Government of the Protectorate

DOI: https://doi.org/10.35622/j.rr.2021.05.009

Recibido: 20-VI-2021 / Aceptado: 28-VII-2021 / Actualizado: 29-VII-2021

\section{Resumen}

La presente investigación se realizó entre los meses de abril y mayo del año 2020, durante la Cuarentena ordenada por el Estado Peruano por la Pandemia de la COVID-19, por lo tanto, sólo se realizó con los libros de la biblioteca personal del autor y en éste trabajo presentamos un estudio del Impacto de la Viruela en las distintas Casas Reales Europeas y cómo el Rey Carlos IV de España buscó prevenir dicha enfermedad a través de la vacuna, tanto en la Metrópolis como en sus dominios de América, incluyendo el Virreinato peruano. La continuidad de la Política Borbónica la veremos en la Normatividad del 1er Estado Independiente: El Protectorado (1821 - 1822).

Palabras Clave: Historia de la medicina, epidemias, Protectorado, Casas Reales.

\begin{abstract}
The present research was carried out between the months of April and May of the year 2020, during the Quarantine ordered by the Peruvian State by the Pandemic of the COVID-19, therefore, it was only carried out with the books of the personal library of the author and in this work we present a study of the Impact of smallpox in the different European Royal

\footnotetext{
${ }^{1}$ Licenciado en Historia por la Pontificia Universidad Católica del Perú (PUCP), egresado de la maestría en historia PUCP; miembro del instituto Riva Agüero PUCP, Instituto Ricardo Palma, rectorado, URP y del Instituto Sanmartiniano del Perú; especialista de historia social sobre Lima del siglo XVIII y mediados del siglo XIX; abordó temas inéditos como los pulperos y pulperías limeñas y la participación de la nobleza titulada durante el régimen del protectorado.
} 
Houses and how King Carlos IV of Spain sought to prevent this disease to through the vaccine, both in the Metropolis and in its domains of America, including the Peruvian Viceroyalty. The continuity of the Bourbon Policy will be seen in the Normativity of the 1st Independent State: The Protectorate $(1821$ - 1822).

Keyword: History of medicine, epidemics, Protectorate, Royal Houses.

\section{ANTECEDENTES HISTÓRICOS}

\section{La Viruela: guadaña de dinastías reales en los siglos XVI al XVIII}

Teofanes Egido sobre la viruela afirmó y no sin razón: "Son bien conocidos los efectos de la epidemia que atemorizaba a todos y que no era tan discriminadora ni respetaba tanto a ricos y privilegiados. En sus ataques no se detenía ni ante las casas reales [...]” (Egido, 2001, p. 200) es por ello que a partir de la bibliografía que poseemos en casa veremos cómo atacó la viruela a soberanos y miembros de dinastías reinantes europeas.

La primera referencia que presentaremos es la del futuro primer soberano europeo del Perú, me refiero al archiduque Carlos de Austria y Trastamara nacido el 24 de febrero de 1500, quien cuando ya había sucedido a su padre el rey archiduque Felipe el hermoso -fallecido en setiembre de 1506- como soberano de los ducados de Borgoña -nominal- Brabante y Luxemburgo condado de Flandes, del Franco Condado, así como de otros territorios en los Países Bajos y ejercía la regencia en dichos dominios su tía la archiduquesa Margarita de Austria; sobre su temprano gobierno el historiador Parker nos dijo que ella: "vivía obsesionada por la salud de los niños dado que como le confió a Maximiliano (el emperador, su padre) incluso la más pequeña enfermedad en personas de tal importancia causa preocupación”. Por tanto, cuando llegó la noticia de que las hermanas del príncipe habían contraído la viruela en Malinas, mantuvo a Carlos en Bruselas "porque los médicos dicen que la enfermedad es contagiosa y mi sobrino podría contagiarse" -como en efecto ocurrió viéndose incapacitado por esta fastidiosa y peligrosa enfermedad durante más de un mes(Parker, 2019, p. 46) el historiador cita para afirmar ello documentos de octubre de 1509 y finales de julio de 1510. Ninguno de los pequeños archiduques infantes falleció y Carlos llegó a ser longevo pues falleció en 1558.

Un segundo caso lo tenemos en Guillermo II príncipe de Orange, Estatúder de los Países Bajos quien el 30 de julio de 1650 mandó encerrar a sus principales críticos en los Estados de Holanda (Parker, 2013) y "de repente el príncipe cayó enfermo de viruela y tuvo que guardar cama, y el 6 de noviembre de 1650 murió de esta enfermedad” (Parker, 2013, P. 418) y como afirmé en un texto "dando al partido descentralizador la oportunidad soñada pues su hijo póstumo no puede disputar el poder a los Estados Generales" (Mera, 2017, p. 175; Adamson y Jonathan, 1999, p. 130). 
En la casa Estuardo, tenemos tres casos, el primero en el hijo menor del decapitado Carlos I, Enrique nacido en 1640, quien había vivido exiliado con su madre en París la reina Enriqueta María de Borbón y retornó a Inglaterra con la restauración de la monarquía en 1660, su hermano mayor el rey Carlos II, le otorgó los títulos de Duque de Gloucester y Earl de Cambridge, pero unos meses después falleció víctima de la viruela (Royal Collection, 2017). Asimismo, Mcleod afirma que la reina Ana tenía el rostro con las huellas de la viruela (Mcleod, 2001) pero su único hijo sobreviviente, el príncipe William, duque de Gloucester falleció en 1700 víctima de la viruela y con ello se extinguió la dinastía, originando la sucesión en la casa de Hannover (Snowden, 2019).

Un quinto caso fue el deceso por viruela del Sacro Romano Emperador José I de Austria a los 35 años de edad, el 17 de abril de 1711 (Wheatcroft, 1995) y un sexto caso, el del zar Pedro II, último varón de la casa Romanov, quien al asistir a la ceremonia de la bendición de las aguas el 6 de enero de 1730 se quejó de dolor de cabeza. Al día siguiente, la presencia de pústulas reveló que padecía viruela, falleciendo el día previsto para su boda a las 3 de la madrugada del 18 de enero (Sebag Montefiore, 2016) no había cumplido aún los 14 años, des Cars nos da el día 19. (Des Cars, 2015).

Los últimos tres casos se presentaron en la casa real francesa. Así, vemos que el 11 de noviembre de 1647 el rey niño de Francia, Luis XIV fue diagnosticado con viruela:

"Durante su enfermedad el niño rey mostró una paciencia y un valor ejemplares, hablando tranquilamente con quienes lo rodeaban, consolando a su madre y no rechazando ningún remedio. Los médicos elogiaron su firmeza, algo sorprendidos de que un niño de nueve años ofreciera el mismo su brazo para que se lo punzaran dominando constantemente el dolor" (Bordonove, 1985, p.51)

De manera que, sobrevivió, pero su hijo Luis nacido en 1661 (Williamson, 1988) y conocido como el gran Delfín, murió de viruela el 8 de abril de 1711, del deceso de su bisnieto que lo sucedió en el trono como Luis XV a los 5 años, y reinó entre 1710 y 1774 (Williamson, 1988) en ese año le dio la viruela y falleció como consecuencia de ella; hemos accedido a una descripción que resumiremos para tener conocimiento de esta terrible enfermedad y que llevó a la tumba al monarca en solo 14 días:

El martes 26 de abril, mientras cenaba con la favorita y el grupo acostumbrado sintió náuseas y no podía tragar... Al día siguiente fue a Saint Denis. Luis tiritaba. De vuelta a Trianon no podía entrar en calor y la fiebre aumentaba. El 28 se declaró francamente enfermo con náuseas violentas... El coche recorrió la distancia de Trianon a Versalles en tres minutos. ... Los médicos de la corte, Bordeu y Leroi llamados de París, consultaron con La Martiniere y estuvieron de acuerdo en la gravedad de la enfermedad, aunque de todos modos no pudieron diagnosticarla. Ninguno de ellos pensó que podía ser viruela, ya que se decía que el rey la había tenido ya en Fontainebleau... Algunos niños hijos de los criados que servían en el Trianon habían muerto recientemente de esta enfermedad. Pero nadie en el areópago médico que rodeaba a Luis XV tuvo la menor idea de que podía tratarse de viruela. Por las dudas se prescribió un emético y una sangría en el brazo. El dolor de cabeza y de riñones, los vómitos y la fiebre no disminuyeron.

Revista Revoluciones -122- Vol. 3, Nº 5 (2021), pp. 120-131

Esta obra está bajo una licencia internacional Creative Commons Atribución 4.0, 
$\mathrm{Al}$ ver esto los médicos ordenaron una segunda sangría más copiosa que la primera en la noche del 29. La enfermedad no quería declararse ...cerca de las diez de la noche hacia las diez y media, en el momento en que los médicos le daban de beber, creyeron notar manchas rojas y exclamaron -acercad la luz: el rey no ve el vaso. Empujándose unos a otros, salieron de la cámara para conferencias volvieron un cuarto de hora después examinaron la lengua del paciente y comprobaron una erupción de viruela... En cuanto al rey, que estaba convencido de haber tenido la viruela en Fontainebleau, no creía tener esa enfermedad. En el momento más intenso de la erupción no vaciló en dejar que Adelaida le tocara las manos, aunque estaban cubiertas de pústulas. Adelaide no demostró la más mínima emoción, a tal punto sabía contenerse. Así mismo dejó que la condesa Du Barry le frotara la frente ... Pero el 3 de mayo a principios de la tarde, el rey se miró las manos purulentas y exclamó de pronto: Es la viruela. El 4 de mayo, cuando volvió el duque de Croy, vio caras consternadas. El duque de Ligne le dijo: La catástrofe es inminente, interrogó a los médicos que le anunciaron que las pústulas habían sido reabsorbidas por lo cual ya no había esperanzas.... Eran las tres de la tarde del 10 de mayo cuando un ujier se presentó en el salón del Ojo de Buey y dijo sencillamente: "El Rey ha muerto" (Bordonove, 1985, p. 314-322)

Así murió el monarca de una de las potencias mundiales de aquel entonces, lo interesante es que este historiador francés nos dice que la familia real francesa no se inoculó, mientras que la mayoría de los príncipes de las cortes extranjeras habían sido inoculados, pero a causa de la hostilidad de la Facultad Luis XV y su familia no se habían vacunado (Bordonove, 1985). Lo que no sucedería con sus primos los Borbones de España.

\section{La casa real española ante la viruela $1788-1806$}

En la familia real española el caso que se recuerde más fue el del infante don Gabriel nacido en 1752 (Williamson, 1988), quien fuese el infante ilustrado por excelencia del siglo XVIII, el más brillante de los hijos de Carlos III y su hijo favorito a quien casó en unas dobles bodas con la infanta portuguesa María Ana Victoria de Braganza, el 12 de abril de 1785, padres de un primer vástago, el infante Pedro Carlos.

el 28 de octubre de 1788 la infanta doña María Ana luego de un laborioso parto dio a luz a un segundo varón el infante don Carlos, pero al quedar extenuada y en el post parto se le presentó un ataque de viruelas malignas que en cinco días le causaron la muerte; no se pudo evitar el contagio del recién nacido y del esposo, muriendo el infantito siete días después de la madre y el infante quince días después de su hijo, el 23 de noviembre (Sainz de Medrano, 1996, p. 7378).

Hechos que no solo llenaron a la corte de luto y que acabó de rematar el corazón de su padre el rey, a quien se oyó decir: "Gabriel ha muerto, Yo le seguiré pronto" (Danvila y Collado, 1923, p. 29) Carlos III falleció en el palacio real de Madrid el 14 de diciembre de 1788. (Fernandez, 2001).

El reinado de Carlos IV como bien afirma Egido (2001): "tenía tristes experiencias por la muerte infantil y juvenil de algunos de sus vástagos, factor que influyó en el interés del rey y

Revista Revoluciones -123- Vol. 3, Nº 5 (2021), pp. 120-131

Esta obra está bajo una licencia internacional Creative Commons Atribución 4.o. 


\section{LA CONTINUIDAD DE LA PREVENCIÓN DE LA VIRUELA EN EL PERÚ: MEDIDAS DEL SUPERIOR GOBIERNO (1806-1820) AL GOBIERNO DEL PROTECTORADO (1822) ISSN: 2710-0499 ISSN-L: 2710-0480}

de sus gobiernos por combatir al enemigo con las novedades que se iban inventando" (p.20o). $\mathrm{Al}$ respecto afirmó que: "Primero fue con la inoculación, medida adoptada ya en la primera parte del siglo XVIII" (p. 200). Y nos dice además que fueron: "sobradamente conocidas las confrontaciones dialécticas que excitó el experimento tan discutido en sus planteamientos teóricos y en los resultados de su aplicación" (p. 200). Egido afirma que fue el propio soberano y no solo su gobierno, el que: "se decantó por lo nuevo y por lo que se creía tan ilustrado, que, hasta el médico de la Junta Superior de Medicina, el célebre Antonio Gimbernat (1734-1816), resultó partidario incondicional. El respaldo del rey se materializó en gestos y en leyes como ha visto Pérez Moreda" (p. 201)

Sigue afirmando Egido que Carlos IV le dio: "El gesto tuvo sus visos de publicidad, si bien estuvo dictado por la precisión de recurrir a lo que fuera con tal de garantizar como fuese la herencia real" (p. 201) y asimismo será que por Egido citando a la Gazeta de Madrid que pudimos saber que a una infanta hija del rey le dio viruela y se recuperó de dicha enfermedad: El 23 de noviembre de 1798 se publicó que la Corte había estaba muy afectada porque la princesa de Parma, la infanta María Luisa -la que sería reina de Etruria por gracia de Napoleón- se vio atacada de viruelas.

Por una investigación que publicamos en el BIRA $\mathrm{N}^{\mathrm{O}} 34$ (2007-2008), hemos dejado constancia, que en la corte de Lima, con motivo de la boda de esta infanta con su primo hermano Luis, príncipe heredero de Parma se mandó hacer una misa solemne en la catedral, el 7 de agosto de 1796 y además hubo tres noches de iluminación en la capital al primer toque de las campanas, a las 7 de la noche de los días 5, 6 y 7 del mismo mes y año (Mera, 2011) con lo cual no quepa la menor duda de que se tenía conocimiento de quién era esta infanta en la población limeña.

En vista de ello nos dice Egido que los Reyes Carlos IV y su esposa la reina María Luisa, nacida princesa de Parma consintieron en la propuesta de sus médicos para librar a sus descendientes del mal que:

tan tristes memorias han dejado a su Familia Real, y no ofreciéndose otro medio que el de la inoculación acreditada por la experiencia y generalmente admitida por las naciones cultas, determinaron someter al sacrificio -aquí debo hacer hincapié que Egido coloca entre paréntesiso a la salvación al príncipe heredero don Fernando (de catorce años) y a los infantes don Carlos María Isidro (de diez años) y don Francisco de Paula (de cuatro años) (Egido, 2001, p.201).

Sabemos también por la investigación que realizamos y publicamos en el BIRA $\mathrm{N}^{\circ} 34$ que la noticia del nacimiento del infante Fernando María, la recibió el cabildo de Lima mediante la cédula dada en Aranjuez el 13 de noviembre de 1784 e inclusive se mandó cumplir la misma en Huancavelica el 15 de julio de 1785 y también se remitió desde Aranjuez la cédula del 20 de abril de 1788 dando conocimiento del nacimiento del infante Carlos María Isidro, durante el reinado de su abuelo Carlos III (Mera, 2011) y respecto del último hijo varón, nació ya siendo soberano y la cédula para que en los reinos de Indias se den las gracias por el nacimiento del infante Francisco de Paula Antonio María, dada en Aranjuez el 28 de abril de

Revista Revoluciones -124- Vol. 3, No 5 (2021), pp. 120-131

Esta obra está bajo una licencia internacional Creative Commons Atribución 4.0. 
1794; dio motivo para que el virrey Francisco Gil de Taboada realizase grandes maniobras en su honor, en los Amancaes de San Bartolomé a extramuros de Barrios Altos, el 3 de setiembre de 1794 a las que concurrió gran número de limeños por lo que tenían pleno conocimiento del benjamín de la familia real inoculado.

La Gaceta de Madrid del viernes 23 de noviembre de 1798 informó de ello y nos decía que: el medio más probable de disminuir los riesgos de una calamidad que casi se ha hecho inevitable y no ofreciéndose otro que el de la inoculación acreditada por la experiencia y generalmente admitida admitida en todas las naciones cultas oído el dictamen de su propio médico de cámara don Francisco Martínez Sobral ...confiando esta delicada operación a la notoria inteligencia y práctica de don Antonio Gimbernat y don Ignacio de la Cava .... Para júbilo de toda la monarquía se hallan en la más perfecta convalecencia ${ }^{2}$.

El resultado positivo de la operación se celebró por todo lo alto con galas misas y Te Deum agradecidos para que todos los españoles se enteraran bien (Egido, 2001). Y así se publicó en la Gaceta de Madrid: Para celebrar mandaron SS MM se vistiese la corte de gala el del corriente (de diciembre) y que en su Real capilla se cantase él Te Deum en acción de gracias al todopoderoso por tan grande beneficio 3 .

Después del experimento real, y por los mismos días se legisló la obligatoriedad de la inoculación en centros públicos de asistencia social, casas de expósitos, de misericordia, hospitales: "a fin de que se adopte generalmente y pueden disminuirse los desastres que causa esta calamidad" (Egido, 2001, p. 201).

Los historiadores que se han ocupado de esta cuestión suelen apostillar que experiencias y leyes llegaban demasiado tarde es decir dos años después de que en Inglaterra el doctor Jenner hubiera dado con el invento decisivo ya de la vacuna, la hermana menor, como se decía entonces, de la inoculación. La posible tardanza, que no fue tanta si atendemos a las posibilidades y velocidades de comunicación -ya que en Inglaterra sucedía lo mismo-, se vio compensada con la aceptación casi unánime de los médicos más prestigiosos, comenzando por los de la Corte, por la acelerada legislación -no siempre cumplida como era de esperarsey con el entusiasmo que convirtió a la Monarquía en promotora de auténticas cruzadas por la salud y contra la viruela. (Egido, 2001).

En España la vacunación se conoció ya en 1799, el gobierno dio normas precisas que legislaron en 1805 la generalización y propaganda de la vacuna mediante una real cédula minuciosa que establecía formularios precisos para los asientos de los vacunados y el análisis y seguimiento de sus reacciones. Se imponía de forma obligatoria en los hospitales bajo responsabilidad del capitán general y se recomendaba que prelados y párrocos persuadieran a sus feligreses a acogerse a la benéfica práctica: "en beneficio de la humanidad y del Estado" (Egido, 2001, p. 202)

${ }^{2}$ Gazeta de Madrid No 94 del viernes 23 de noviembre de 1798, pp. 1004-1005.

3 Gazeta de Madrid No 94 del viernes 23 de noviembre de 1798, p. 1005.

Revista Revoluciones $\mathbf{- 1 2 5}$ - Vol. 3, No 5 (2021), pp. 120-131 
Sería el cirujano del rey Francisco, Javier de Balmis (1756-1819) quien formuló de manera mucho más ambiciosa en su proyecto de un periplo que recorrería todas las indias, las occidentales y las orientales de la Monarquía española predicando las excelencias de la vacuna que justamente el 1802 , acababa de azotar vastos territorios de aquellas posesiones encontramos noticias de inoculación de la vacuna publicada en la Gaceta sobre Andújar en octubre de $1802^{4}$ y bajo el título de América Española sobre México y Puebla ambos de página y media de extensión que narran los hechos de octubre de 1804 publicados en junio de $1805^{5}$.

\section{El Virreinato Peruano y la vacuna contra la viruela 1806-1820:}

Afirma Lastres que el arribo de la Real Expedición Filantrópica de la Vacuna a nuestro país significó un gran acontecimiento médico-social y un estímulo para la decadente medicina de principios del siglo XIX Lastres inclusive afirma que pudo alentar a Unanue a crear su obra magna, el Real Colegio de Medicina y Cirugía de San Fernando (Lastres, 1957). La Junta Conservadora del Fluido Vacuno se estableció por un decreto superior del virrey Fernando de Abascal y Souza el $1^{\circ}$ de julio de 1806 , la que funcionó de forma periódica hasta 1820 . Como bien hace en afirmar Lastres aquella Junta fue el primer organismo técnico en orden a la conservación de la Salud Pública en el virreinato del Perú y como veremos se lograría una continuidad durante los primeros años tan convulsos de vida independiente como lo fueron los años entre 1822 y 1825 .

La Junta General de Conservación y Propagación del Fluido Vacuno estuvo constituida en 1820 por los siguientes miembros, el presidente era el Excmo. Sr. Virrey Pezuela, el copresidente, el Excmo. e Ilmo. Sr. arzobispo de Lima, el vicepresidente Francisco Xavier Moreno, Vocales, el Sr. alcalde, el Sr. Regidor Decano, Sr. Dn. Carlos Orbea, Sr. Dn. Joaquín Bonet, Sr. Dn. Pedro Juan Sanz, Sr. Dr. Dn. Miguel Tafu, Sr. Dn. Martin Aramburú, secretarios con voz y voto Dn. Ignacio Mendizábal, Dn. Juan Bautista Valdeavellano, Médicos consultores con voz y voto informativo D. D. José Manuel Dávalos, DD. Félix Devoti, Porteros del Ayuntamiento.

En 1820 murió Dávalos y lo sucedería en el cargo a partir del siguiente año de 1821 el Dr. José Manuel Baldes (p. 172-173). Así mismo nos dice Lastres que el 19 de mayo de 1820 terminaron las labores de la primera Junta Conservadora de Fluido Vacuno en lo que a sesiones regulares se refería y era evidente que la viruela había aminorado su epidemicidad y solo de vez en cuando se mostraba bajo la forma de brotes (p. 173-174). Este cese se dio durante el mandato del virrey Joaquín de la Pezuela, tres meses y días antes del desembarco de la Expedición Libertadora en San Clemente de Pisco.

El médico José Manuel Valdés definió a Lima como de "temperamento caliente y húmedo" y que sus habitantes no son tan vigorosos como los que viven en la sierra. Por eso tienen

4 Gazeta de Madrid No 80 del viernes $1^{\circ}$ de octubre de 1802, p. 987.

5 Gazeta de Madrid No 45 del martes 4 de junio de 1805, pp. 484-486 y No 46 del viernes 7 de junio de 1805, pp.496-498. 
predisposición a padecer "enfermedades inflamatorias" sobre todo en las épocas de transición de calor al frío y viceversa. En el año 1818 hubo una epidemia biliosa pútrida de evolución a veces mortal y afirma que cuando entraron las tropas del General San Martín también había una de estas epidemias pero que no eran de viruelas. Lastres también afirmó que, del análisis cuidadoso de los documentos insertos en las obras de Jerónimo Valdés, Torrente y otros del lado realista no indicaron que la viruela haya dificultado la marcha de los ejércitos al interior del país.

\section{El Protectorado y la normatividad para la vacuna contra la viruela de 1822}

Si bien el Protectorado surge como forma jurídica el 3 de agosto de 1821, será recién durante el mandato del Supremo Delegado marqués de Trujillo, en nombre del Protector del Perú, que se emitirá una norma al respecto el 16 de febrero de 1822 y en sus considerandos se dijo lo siguiente: "al mencionar que la viruela era una plaga que desde el centro de la Arabia, se ha esparcido por la superficie del globo ha desolado más la especie humana que todas las guerras causadas por las disputas entre el trono y el altar y entre los reyes y los pueblos. Jenner el hombre inmortal que descubrió el antídoto de aquella ha cerrado más sepulcros que cuantos han abierto los Conquistadores en el de sus empresas (Oviedo, 186o, p.321; Lastres, 1957, p.176) el decreto que también fue firmado por el ministro Bernardo Monteagudo especificaba la forma como se ha de proceder para evitar la viruela:

1. Todos los curas antes de salir de sus curatos se presentarán ante el Protomédico Dr. Miguel Tafur de quien recibirán el fluido vacuno debiendo exhibir ante el Presidente del Departamento (en aquel entonces era José de Riva Agüero Sánchez Boquete presidente. del departamento de Lima) el certificado de haberlo así cumplido antes de obtener el pasaporte. En seguida ocurrirán al ministerio de Estado a recibir el número de ejemplares que según la extensión de las parroquias necesiten del método para aplicar la vacuna que se han mandado imprimir de cuenta del gobierno.

2. Cada mes darán razón a los presidentes de los departamentos al que corresponden sus doctrinas del número de niños que hubiesen vacunado, para que aquellos las remitan al ministerio de Estado.

3. Señalarán un día de cada semana para que se reúnan los niños que deben ser vacunados, compeliendo a las madres a que los lleven y consultando por este medio que ni sean todos vacunados (sic) en un día ni falte jamás el número necesario para la propagación del virus.

4. Igual obligación tendrán los tenientes de curas en las vice-parroquias y anexos de cada doctrina.

5. Los presidentes, gobernadores y tenientes gobernadores nombrarán en su distrito un facultativo que se distinga por su patriotismo y filantropía con el título de Inspector de Vacuna para que ayude a los párrocos en esta interesante comisión.

6. Los prelados de los conventos de regulares en todo el territorio del Estado, nombraran igualmente aquellos religiosos que consideren más aptos y celosos (sic) para que un

Revista Revoluciones -127- Vol. 3, N 5 (2021), pp. 120-131

Esta obra está bajo una licencia internacional Creative Commons Atribución 4.0. 
día de cada semana se empleen en vacunar(sic) a los niños debiendo comisionar para esto al menos la tercia parte del número de los individuos que tenga cada convento.

7. Los prelados remitirán mensualmente al ministerio del Estado la razón de los niños que se vacunasen en sus conventos, para que se publique con los demás a que se refiere este decreto.

8. Los comisarios de barrio en las ciudades donde las hay y los gobernadores o tenientes gobernadores de conservación y propagación del fluido vacuno (sic) en esta capital y en los demás pueblos a sus respectivos párrocos para que se obligue sin demora a las madres a presentar a sus hijos en los términos que se halla prevenido. Todo hombre sensible al bien de sus semejantes queda encargado de la ejecución de este decreto que se insertará en la Gaceta Oficial6 (Oviedo, 1860, p. 322; Lastres, 1957, p.177).

El Doctor Tafur presidia el protomedicato y afirma Lastres que: "hace algunos años que ha tomado partido por la vacuna jenneriana" declarando ser "el descubrimiento más importante a la humanidad" y "ordena que los curas se presenten ante el a recabar el certificado de haber recibido la vacuna para propagarlo en su respectiva provincia" (Lastres, 1957, p. 178). Aquí encontramos una nota a pie de página en el capítulo pertinente de Lastres que consideramos muy importante para esta nota pues implica la primera publicación de medicina preventiva durante el Protectorado y del Perú independiente: El método de Moreau de la Sarthe traducido por Balmis para aplicar la vacuna. Lastres opina como médico sobre este decreto y nos dijo al respecto:

Seguramente que este decreto con ser bueno desde el punto de vista humanitario no lo es si lo vemos del lado estrictamente técnico pues entrega la vacuna a manos empíricas como en la de los prelados. Aunque el procedimiento es sencillo no dejaba de tener inconvenientes para personas no técnicas. Seguramente Tafur tuvo en mente la escasez de facultativos en las provincias y por eso delegó en los párrocos esta función, la que duraría algunos años. Con todo, dada la alta capacidad de los siervos de Dios, no dudamos que las disposiciones se llevaron a cabo con puntualidad y que todo ello fue en bien de la medicina preventiva. Por ello es que se habla muy poco de la viruela durante las campañas emancipadoras (Lastres, 1957, p. 178).

Así mismo Lastres cita un valioso documento que ubicó en el Archivo Ministerio de Hacienda, el "Expediente iniciado sobre el precioso fluido vacuno, 1822" en el cual se relata el esfuerzo de las autoridades del gobierno Protectoral para propagar el fluido, cuando José de la Riva Agüero, era presidente del departamento, este afirmó que se vacunaba activamente en la capital y en pueblos del interior: Los comisarios de Barrio reúnen a los niños para que reciban la vacunación. Nicolás Bezanilla con fecha 2 de marzo de 1822, dirige una comunicación en este sentido. Da cuenta de que se han vacunado 69 individuos: "en mi Barrio a quienes no les ha dado la viruela en cumplimiento del Supremo orden de ejecutarlo así todos los meses los comisarios. Muchos se oponían a la sencilla operación y el Comisario va de casa en casa para indagar si se ha llevado a cabo la inoculación decía que algunos niños no concurrían al

${ }^{6}$ Gaceta de Gobierno Tomo $2{ }^{\circ} \mathrm{N}^{\mathrm{O}} 14$.

Revista Revoluciones -128- Vol. 3, No 5 (2021), pp. 120-131

Esta obra está bajo una licencia internacional Creative Commons Atribución 4.o. 
Ayuntamiento, como se empezó a denominar al antiguo Cabildo virreinal, por vivir demasiado lejos y por estar andrajosos.

Por ello Bezanilla opinó que debería de realizarse la inoculación en la casa de los Comisarios y decía: "observando la alternativa por Cuarteles, Barrios, entre dos Facultativos encargados según el método que para ello se estableciese" (Lastres, 1957, p. 179). El comisario tendría la misión de "traerles los párvulos para propagar el pus vacuno" (Lastres, 1957, p. 179). Lo interesante es que el presidente del Departamento Riva Agüero le contestó al Comisario Bezanilla, el 14 de marzo de 1822, no estar de acuerdo con su opinión y más bien creía que para evitar las viruelas:

Se puede resolver que el médico rote por las casas de todos los Comisarios, principiando por el barrio $1^{\mathrm{o}}$ del Cuartel $1^{\mathrm{o}}$ y sucesivamente por los demás cuarteles hasta que concluya el quinto quedando a cargo del Juez de cada uno avisar al Comisario que le toque el turno para que concurra a su casa y de esta lleve al médico a lo que tenga en el barrio de su pertenencia, cuya molestia se hará tolerable al operante considerando el gran beneficio que de ella resulta a sus semejantes. Sobre todo dispondrá de V.S.Y. lo que le parezca más conveniente en obsequio a los miserables (Lastres, 1957, p. 179).

El Dr. Manuel Muelle, abogado de la Alta Cámara da cuenta del acto celebrado en la Municipalidad y concluye que:

"siendo dos los profesores de la Vacuna, uno de ellos continúe asistiendo en la Sala destinada al efecto y que rotando por los barrios respectivos; esperando el Cabildo que esta molestia se le hará tolerable al operante considerando el beneficio que de ella resulta a la humanidad; a cuyo efecto se hiciere saver (sic) esta acta a los expresados profesores con copia certificada. El contenido de esta acta se hizo de conocimiento a los médicos consultores Valdés y Devoti” (Lastres, 1957, p. 180).

Finalmente debemos de señalar que José Manuel Valdés desempeñó el papel de vacunador y también médico de los presos de la cárcel, presentó a la Municipalidad un reclamo debido a que todo ese año de 1821 estuvo trabajando solo, ya que su compañero el Dr. Devoti se encontró empleado en la Tesorería del Estado y que aquel había percibido 50 pesos mensuales, mientras que a él solo después de muchas súplicas le dieron en el mes de junio de 1822, 100 pesos, no se le había abonado su sueldo de médico de los presos de la cárcel, que había donado un mes de su sueldo a la patria, es decir 50 pesos y se le debía 600 pesos. El gobierno republicano reconoció ser justa su petición, reconociendo estar atrasados en los fondos públicos para satisfacer la deuda de Vacuna del año pasado de 1822.

Cuando ya a través de un golpe de estado se había proclamado la República, Valdés dejó constancia que el 14 de marzo de 1823 había asistido a la vacunación en la Sala Capitular de la Municipalidad pero nadie concurrió a vacunarse en aquella tarde a pesar de haberse puesto desde la mañana la bandera, que es el signo convocatorio, según sus palabras: "Dicha falta, muy sensible pudo ocasionar a su juicio, la pérdida del fluido” (Lastres, 1957, p. 180), sobre

Revista Revoluciones -129- Vol. 3, № 5 (2021), pp. 120-131 
Valdés en un trabajo biográfico que escribió Lastres en 1937 nos dice que llega a poseer el preciado título ya en la cuarentena de la vida retemplada su alma en la dura lucha del trabajo y acibarado su espíritu por las mezquindades de una sociedad reaccionaria. A partir del año 1807 se abren para Valdés todas las puertas sociales. Está también en el apogeo de su poderío intelectual y continúa afirmando que su clientela había aumentado considerablemente.

Aunque alejado un tanto del cultivo de las ciencias por esa pasión por la política que todo lo absorbe no tenía tiempo de dedicarse a la clientela o a la Clínica. Por otro lado, había tenido que ausentarse para hacer viaje a Europa. Valdés rápidamente se granjeó la simpatía social y formó una numerosísima como selecta clientela. Además, era médico de los hospitales de San Pedro y de San Juan de Dios, así como de varios monasterios. Actividad polimorfa la del sabio cuyos pocos momentos de reposo que tenía los dedicaba al culto de las musas, pero ellas le deberían de recompensar con creces y será recompensado durante el Protectorado pues “ingresó a la Sociedad Patriótica fundada en 1822. Ese año el Gobierno de Torre Tagle cuando ejercía como Supremo Delegado- lo nombró médico de Cámara del Gobierno equivalente al antiguo cargo de Médico de Cámara del Rey. Además, es condecorado con la cruz de asociado de la Orden Nacional del Sol del Perú.

\section{CONCLUSIONES}

Los antecedentes históricos que hemos podido ubicar nos permiten entender la letalidad que la viruela tuvo en las dinastías europeas y a su vez permite entender la política adoptada por el rey de España de aquel entonces y consideramos válida para la historia de la medicina del Perú; la afirmación de Egido que: "El logro más espectacular y más decisivo de toda la política de Carlos IV fue el de la vacuna contra la viruela" (Egido, 2001, p. 200) y como hemos visto, ello redundó en sus dominios de las Indias y por ende en el virreinato peruano y a ello le agregamos que los pocos médicos peruanos eran de dotes extraordinarias y junto con la autoridad del protomedicato que juró y firmó estar de acuerdo con la Independencia el 29 de julio de 1821 hizo como bien señala Lastres que: Los médicos Hipólito Unanue y Miguel Tafur fueron los consejeros de ambos Libertadores y el decreto sobre la propagación del fluido vacuno del 16 de febrero de 1822 fue una prueba elocuente de ello, sin dejar de lado el intachable desempeño del doctor Juan Manuel Valdés y las recompensas que recibió durante el Gobierno del Protectorado.

\section{REFERENCIAS BIBLIOGRÁFICAS}

Bordonove, G. (1985) Los Reyes que hicieron Francia Luis XIV, el rey sol. Buenos Aires: Javier Vergara Editor, Tomo 3.

Bordonove, G. (1985). Los Reyes que hicieron Francia Luis XV. Buenos Aires: Javier Vergara Editor, Tomo 4.

Danvila y Collado, M. (1923). Historia del reinado de Carlos III, En Historia General de España escrita por individuos de nuevo de la Real Academia de la Historia. Madrid: Edit. Progreso, Tomo VI.

Revista Revoluciones -130- Vol. 3, Nº 5 (2021), pp. 120-131

Esta obra está bajo una licencia internacional Creative Commons Atribución 4.o. 
Des Cars, J. (2015). La Saga de los Romanov. Buenos Aires: Editorial El Ateneo.

Egido, T. (2001). Carlos IV. Madrid: Arlanza Ediciones.

Fernández, R. (2001). Carlos III. Madrid. Arlanza Ediciones

Israel, J. (1999). The United Provinces of The Netherlands. The courts of the house of Orange

c. 1580-1795. En John Adamson, The Princely courts of Europe Ritual Politics and Culture under the ancient regime 1500-1750. London: Widenfeld \& Nicolson.

Lastres, J. (1937). El doctor Juan Valdes. Revista Médica Peruana, 1(106).

Lastres, J. (1957). La salud pública y la prevención de la viruela en el Perú. Lima.

Macleod, J. (2001). Dynasty The Stuarts 1560-1897. New York: St. Martin press.

Mateos Sainz, R. (1996). Los desconocidos infantes de España Casa de Borbón. Barcelona, Thassalia.

Mera, A. (2011). Efemérides dinásticas de los borbones en la corte de Lima. BIRA.

Mera, A. (2017). Cronología de los Países Bajos (1548-1689). En Neerlandeses en América Latina (Rosas y Ortiz eds.) Lima: Fondo Editorial URP.

Oviedo, J. (1860). Colección de leyes del Perú.

Parker, G. (2013). El siglo maldito, Clima, guerra y catástrofes en el siglo XVII. Barcelona: Planeta.

Parker, G. (2019). Carlos V. Una nueva vida del emperador. Barcelona: Editorial Planeta.

Royal Collection Trust. (2017). Charles II Art \& Power. London: York House St James Palace.

Sebag, S. (2016). Los Romanov, 1613-1918. Barcelona: Editorial Planeta.

Snowden, F. (2019). Epidemics and Society from the black death to the present.

Williamson, D. (1988). Debretts Kings and Queens of Europe. Boston: Salem House Publishers.

Wheatcroft, A. (1995). The Habsburgs Embodying empire. New York: Viking.

\section{FUENTES PRIMARIAS}

Gazeta de Madrid (1798)

Gazeta de Madrid (1802)

Gazeta de Madrid (1805)

Gaceta de Gobierno de Lima (1822) 